\title{
Reservoir assessment using non-invasive geophysical techniques
}

\author{
Philippe Sentenac $^{1} \mathbb{D} \cdot$ Vojtech Benes $^{2} \cdot$ Helen Keenan $^{1}$
}

Received: 22 May 2017 / Accepted: 31 March 2018

(c) The Author(s) 2018

\begin{abstract}
This paper describes the use of three geophysical techniques to detect potential seepage that could jeopardise the integrity of reservoir embankments, could induce partial or total collapse and pose a risk to the population nearby. A fast-scanning geophysical technique using two dipole electromagnetic (EM) profile apparatus GEM2 provided the first step to detect the weakest points on the selected dams in order to proceed to a more detailed analysis and visualisation of the soil erosion (fissuring or piping) using electrical resistivity tomography (ERT). Finally, self-potential surveys were carried out to relate to the EM and ERT anomalies that could be pathways for seepage and changes of the water displacement inside the embankment. The three geophysical techniques used were evaluated in one case study of reservoir in a location relevant to flooding issues in Czech Republic. A risk approach based on the geophysical results was undertaken for the reservoir embankment. The three techniques together were compared for the same problematic section and confirmed seepage by showing similar results. Conclusions were also drawn on the efficiency of using these three techniques as a package to give a comprehensive non-invasive assessment to be used as common practice by local authorities and environment agencies whereby remedial action could be recommended to protect assets and civilians.
\end{abstract}

Keywords Reservoirs $\cdot$ Dam assessment $\cdot$ Geophysics $\cdot$ Seepage

\section{Introduction}

When coupled with the predicted increase in precipitation, the importance of maintenance and inspection of reservoir dams is of increasing public importance in the UK. Currently, the common approach to assess reservoir embankments is by visual surveying, requiring the surveyor to walk the entire length of the structure to observe any possible weaknesses. The presence of defects such as erosion and burrows can be obscured by dense vegetation, and as a result, surveys are generally taken during the winter months when vegetation is lighter. Despite this, vegetation can still be heavy enough to impede the visual survey particularly on the landward side which is rarely maintained. The severity of the erosion inside the structure can be underestimated

Philippe Sentenac

philippe.sentenac@strath.ac.uk

1 Department of Civil and Environmental Engineering, University of Strathclyde, John Anderson Building, 75 Montrose Street, Glasgow G11XJ, UK

2 G IMPULS Praha s.r.o., C/28073, Nerudova 232, 25261 Jeneč, Czech Republic due to seasonal swelling of the soil during wetter months. In addition to visual surveys, only destructive techniques, such as sampling and trenching, can be used to detect erosion in depth (Dyer et al. 2009).

Hence, new monitoring approaches are needed which would allow non-invasive detection and characterisation of erosion and seepage in the near surface. Modern geophysical surveys often require the use of two or more complimentary techniques in order to verify the locations of anomalies (Hadley 1983; McDowell et al. 2002; Nguyen et al. 2005; Reynolds 2011) and coupled with geotechnical analysis to confirm findings (Weller et al. 2014; Bièvre et al. 2017). For instance, S-wave velocity and resistivity or P-wave refraction surveys are used to classify the soils used for river embankment (Takahashi and Yamamoto 2010) or to follow fluid-induced variations (Bergamo et al. 2016). A combination of seismic and electric resistivity can locate potential seepage locations in an embankment (Chao et al. 2006). Looking at the seismic noise can allow monitoring of the internal erosion of the embankments in a time-lapse manner (Planès et al. 2016). Other geophysicists preferred the use of ground-penetrating radar (GPR) for the monitoring of river embankments (Di Prinzio et al. 2010) and detection of 
seepage, combined with drill testing (Antoine et al. 2015). Since air-filled voids provided an excellent dielectric constant contrast, GPR revealed to be suitable for identifying animal burrows in earthen embankments and levees. The GPR technique is an extensive investigation method that enables one to rapidly cover a wide area, locating voids, and it is easy to use with the possibility to inspect the collected data in real time. Nevertheless, GPR analysis needs expertise and modelling skills and embankments are various in natures and often made of conductive materials, such as clay, rendering the GPR survey very limited. It is more and more frequent to see in the literature reviews some evaluations of a full set of geophysical techniques in order to assess their suitability. For example, Fauchard and Meriaux (2007) wrote guidance on geophysical and geotechnical methods for diagnosing flood protection dikes. (Niederleithinger et al. 2012) assessed resistivity, electromagnetic, seismic, and GPR techniques at a test site along the Mulde River in eastern Germany, giving advantages and inconveniences of the techniques reviewed.

An examination of the literature discussing geophysical techniques applied to surveying flood embankments have shown the use of electrical resistivity tomography (ERT) to be common, due in part to the suitability of the construction materials (normally compacted, fine grained soils) and its sensitivity to changes in moisture as well as its ability to detect discontinuities in the material (Fargier et al. 2014; Seokhoon 2012). ERT has been found as a good nondestructive technique, which gave the possibility of detecting internal erosion processes and to detect anomalous seepage (Sjödahl et al. 2006, 2010; Cho and Yeom 2007; Lin et al. 2014), at an early stage before the stability of the dam was compromised. In association with ERT, geophysical techniques such as electromagnetic scanning and self-potential have been suggested as non-invasive methods for examining internal embankments conditions (Sentenac et al. 2013, 2017; Jones et al. 2014; Utili et al. 2014; Ikard et al. 2014; Bolève et al. 2011). For instance, repeated geoelectrical measurement (SLINGRAM, ERT, SP) during the dry season and flood event was used for more precise interpretation of the seepages through embankment and dams (Benes et al. 2011). Electromagnetic Slingram methods involving lowfrequency equipment (GEM-2, CMD, and EM31) for scanning embankments are far the fastest techniques (Viganotti et al. 2013; Sentenac et al. 2013) with GPR (Perri et al. 2014). Others, like Mydlikovski et al. (2007) used high-frequency electromagnetic methods: The depth of penetration of electromagnetic waves was small due to the fact that, in this medium, electromagnetic waves are strongly attenuated, and the structural weaknesses of levee caused by high level of ground water is characterised by high level of electrical permittivity and low resistivity. For fast detection of seepage inside embankments, the self-potential (SP) technique has been the preferred one (Rittgers et al. 2015; Bolève et al. 2011; Minsley et al. 2011), and salt tracers injection have also been used with SP to enhance the detection of preferential flow path in heterogeneous media (Ikard et al. 2012).

The potential of the conventional electrical, electromagnetic, and self-potential methods to detect severe erosion and to confirm seepage was assessed against one reservoir in South Bohemia (Czech Republic).

\section{Methodology}

The three complementary geophysical techniques were used to scan the reservoir dam following the same methodology and procedure.

\section{Electromagnetic surveying}

Electromagnetic surveys (EM) were undertaken in order to rapidly scan the entire reservoir dam. The results are based on the measurement of the soil conductivity (inverse of the resistivity) over a measured section and have the advantages of being rapid and contactless. Electromagnetic measurements were used to identify possible locations of the embankment presenting structural defects, such as piping or the presence of drains or cavities if they are fully filled or partially filled with conductive materials (clay, water). The method can detect high fluctuations in conductivity.

\section{Principle}

The electromagnetic profiling is based on the measurement of induction of the primary electromagnetic field of the transmitting coil in the surrounding investigated medium. The primary field induces a secondary field in the ground whose intensity depends on the conductivity (resistivity) of the medium surrounding the transmitting coil. Therefore water ingress can be detected.

The equipment used for the surveys was the GEM2 (GEOPHEX USA). The measurements obtained from the electromagnetic survey were based on the evaluation of induced secondary magnetic field. The transmitter generates pure sine wave of magnetic field with vertical/ horizontal dipole orientation. The receiver with the same dipole orientation is placed on the arm with proper length with the respect to the nominal depth range. The received secondary magnetic field consists of an imaginary part (out of-phase) which is proportional to apparent electrical conductivity when assumption of low induction is fulfilled and calibrated in $\mathrm{mS}$ and of real part (in-phase) which is determined by magnetic properties (significantly influenced by ferromagnetic objects) and shown in part per thousand (ppt) of the primary field. The respective 
frequencies were $6525,13,025,27,025$, and 47,025 Hz. They were carried out "continually" at a walking pace. The results density was approximately 3-4 scans per $1 \mathrm{~m}$ of profile. The device was connected to GPS navigation, hence recording the measurement positions automatically. The measured data from GEM2 were analysed with the programmes DIKINS analyser (MEASProg-Czech Republic). The DIKINS analyser is a software for preparation of resistivity (and other parameters) graphs, map of measured profiles, and interpretation of measured data. Interpretation of measured data means extent of quasihomogeneous blocks and parameter "risk factor" which show the potential zone at risk on the embankment (material inhomogeneity, materials with higher permeability (resistivity), unknown artificial structures, etc.). The output is a csv data file with measured conductivity data and their interpretation, which can be used for construction of GIS layer.

The electromagnetic Slingram method (EM) provided significant advantages for shallow environmental characterisation. Unlike seismic or ground-penetrating radar methods that involve heavy logistics and labour-intensive field work, the GEM-2 apparatus requires only a single operator, is contactless with the ground (thus, is less intrusive), and can operate at a stand-off distance. The relative high-frequency signal can travel only a short distance and thus, "sees" only shallow structures.

The depth to obtain the information on the conductivity of the medium depends on the frequency of the primary electromagnetic field or on the length of the transmitter coils. High frequencies reach lower depth penetration than low frequencies. Based on the normalised sensitivity curve of each instruments, for the CMD2 from Gf instruments the effective depth corresponds to the curve where $75 \%$ of the cumulative sensitivity is reached. The depth penetration of the GEM-2 is dependent on the coil orientation and on the frequency. The GEM- 2 can record up to 15 frequencies simultaneously. The frequency band within which the GEM-2 operates is $330 \mathrm{~Hz}-48 \mathrm{kHz}$. Higher frequencies generally respond to shallower parts of the ground than lower frequencies, so using a range of frequencies means that a greater range of depths is sampled than would be using a single frequency. However, the resistivity of the ground limits the depth to which any EM instrument can 'measure'. Geophex estimate the GEM-2 should be able to see about $20-30 \mathrm{~m}$ in resistive areas and about $10-20 \mathrm{~m}$ in conductive areas. The more conductive the ground, the shallower the GEM-2 can measure; for very conductive ground conditions the depth at which the lower frequencies sense is greater than the depth to which the EM radiation can penetrate, and the lower frequencies are effectively blinded (Reynolds 2011). The GEM-2 can exhibit various results at various frequencies if the soil contains magnetic materials, and then the device is sensitive to magnetic susceptibility and viscosity (Simon et al. 2015); this is not the case in the present study.

\section{Resistivity surveying}

Electrical resistivity tomography (ERT) is a well-established geophysical technique that is commonly applied at the ground surface and has particular promise in complementing conventional methods of site investigation towards spatially improved characterisation of subsurface water, soil layers and pollutant transports (as reviewed e.g. in Slater 2007). Aside from delivering improved spatial information, in situ ERT surveys are also rapid and cost-effective compared to traditional methods of access-hole sampling and analysis.

The resistivity array technique is routinely used to map ground water and contaminated flows, and it is also regularly used to locate buried artefacts or structures. ERT performs particularly well in soil media consisting of clay or silty soils due to the excellent contact between electrodes and the soil. The high concentration of charged particles in the soil also facilitates electrolytic conduction which is necessary for the ERT to perform well. Modern resistivity surveys involve the use of computer-controlled multi-electrode arrays in order to efficiently obtain resistivity measurements over the surveyed section. The inversion of this data with software like Res2DInv or Res3DInv (GEOTOMO Software-Malaysia) gives a tomography contour model of the subsurface in two or three dimensions (Griffiths and Barker 1993).

The principle of the method is based on the measurement of the soil apparent resistivity, using a large number of electrodes placed along the profile or in the area. The electrodes are interconnected by a special cable that enables to connect the electrodes as current ones and potential ones step by step. This allows performing the measurement for a large number of variants of a four-electrode array with differing geometry and penetration depth. The measurement proceeds automatically; everything is controlled by a PC. For the resistivity tomography measurements, the device ARES (GF Instruments, Czech Republic) was used. The distance between the electrodes was $2.5 \mathrm{~m}$ on longitudinal profiles of the embankments and $1 \mathrm{~m}$ on the perpendicular profiles. $2 \mathrm{D}$ resistivity sections were compiled using the programme Res2DIinv. A Schlumberger electrode arrays configuration was chosen for the ERT measurements. The results were collected using the fifth iteration of least squares method.

\section{Self-potential method}

The self-potential method (SP) measures the natural electrokinetic potential of soils and rocks using non-polarisable $\mathrm{Cu} / \mathrm{CuSO}_{4}$ electrodes filled with a copper sulphate solution. This method is a passive technique. In dam surveying, the filtration potential produced by water through 
a porous medium can often be detected. The principle of this phenomenon consists in unequal mobility of anions and cations transferred by the liquid medium through the porous material. This inequality generates measureable negative potential at the point of infiltration and positive potential at the point of outflow. When interpreting SP potentials, it is necessary to distinguish a natural filter electric field from interfering fields caused by the natural electrochemical process or artificial fields (for example, near railways, cables and tubes). The outcomes of the measurement using the SP method are profile curves and graphs of electric potentials. Based on their analysis, the locations of potential seepage paths through the levee can be identified. To assess the presence of seepage, it is convenient to take measurements on the land side during dry season (decreased water level in the reservoir) and during the flood (maximal water level in the reservoir). The method is suitable also for repeated monitoring measurements aiming to detect long-term changes in the seepage regime of the levee and its underlying layers.

The measurements in Czech Republic were performed with a homemade $\mathrm{mV}$ meter with the capability of SP measurements. Two non-polarised $\mathrm{Cu} / \mathrm{CuSO}_{4}$ electrodes were also used for SP surveys. The electrodes were placed together at the soil surface to zero the potential. One electrode was set as reference, and the other was used to take the potential inside the soil. Measurements were taken every $5 \mathrm{~m}$ over the length of the dam.

\section{Surveys results}

\section{Czech reservoir Vitineves}

A site map indicating the location of the Czech geophysical surveys is presented in Fig. 1. The site was selected as seepage was suspected from several field observations.

The Vitineves reservoir is located $5 \mathrm{~km}$ to the south of the town of Jičín in central Bohemia. It is a small water reservoir built in the second half of the twentieth century.

The dam was built with local materials made of silty and sandy clay and loam. Figure 2 shows the type of local materials available at the proximity of the reservoir location represented by the red cross on the geological map of the area.

The reservoir was designed to capture water draining from surrounding fields and is now used mainly for recreational purposes (fishing). The dam is formed by an earth body with a length of $150 \mathrm{~m}$ and a maximum height of $3 \mathrm{~m}$ (Fig. 3).

The aim of the geophysical survey was to assess the material homogeneity of the dam and to determine the extent of seepages occurring. The results were used to design remedial measures to reduce seepages. The survey was conducted

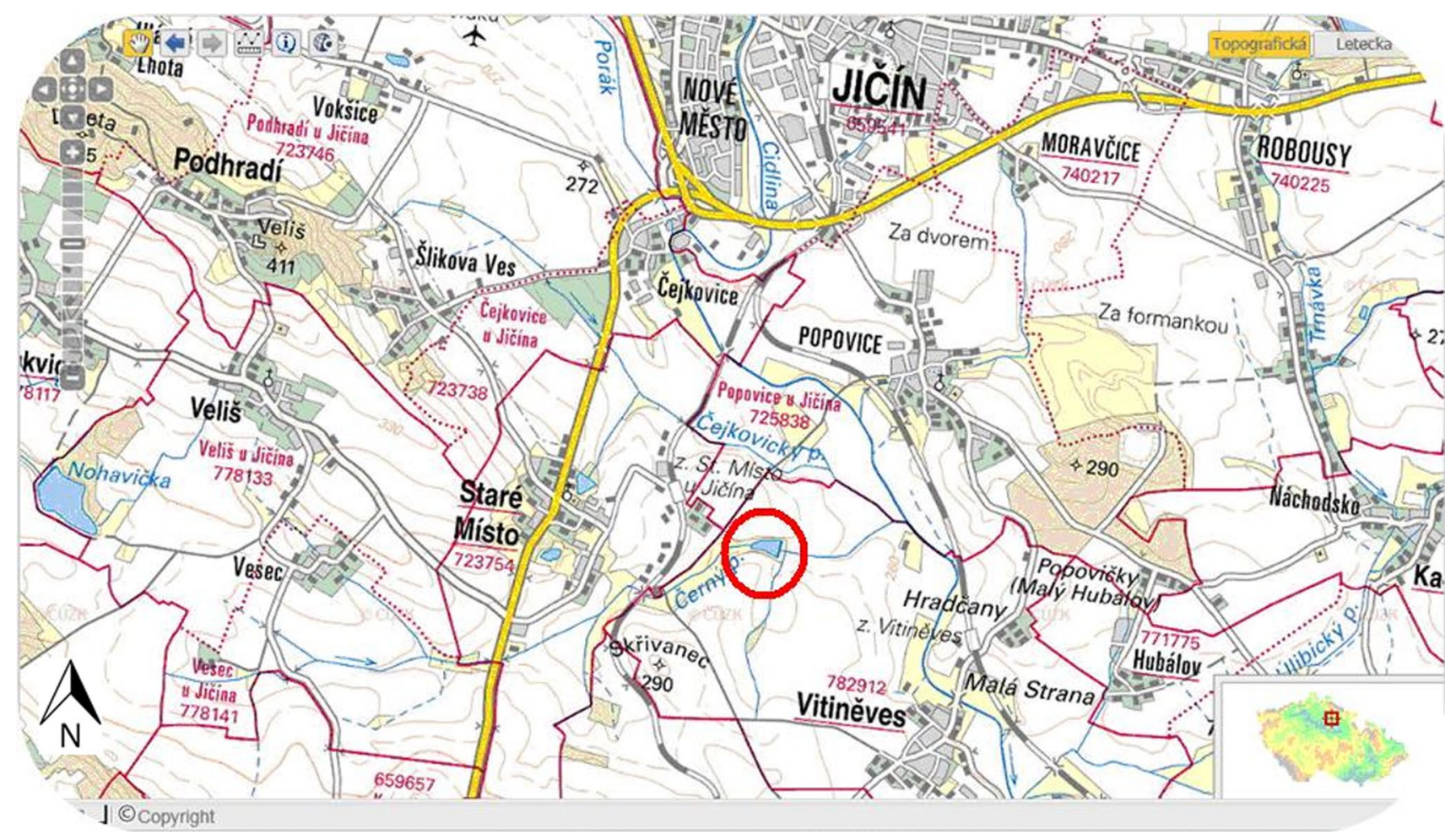

Fig. 1 Location of the Vitineves reservoir (Czech Republic) 


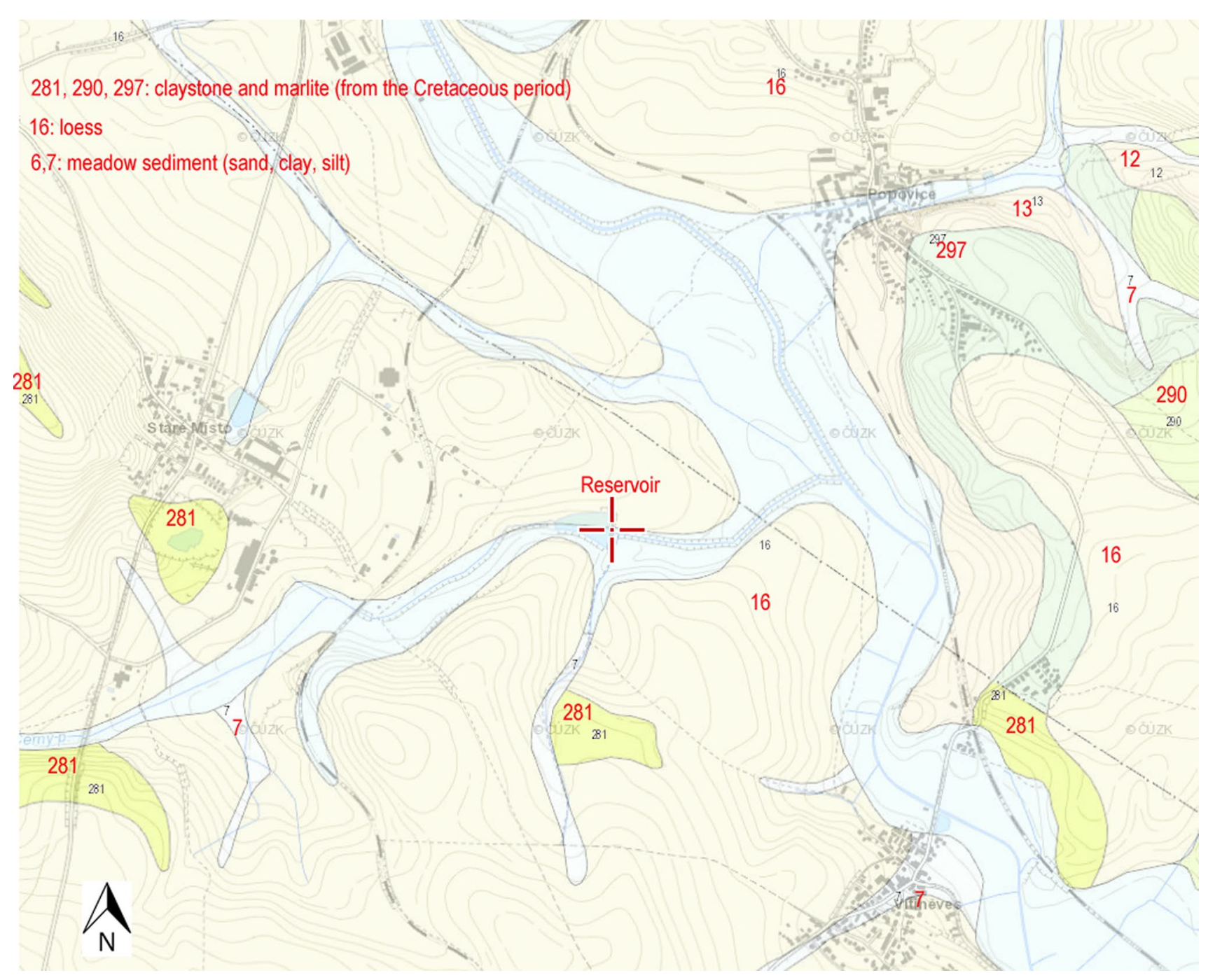

Fig. 2 Geological map Vitineves area

using the above-described methods (Slingram, ERT, and SP) in two stages. The GEM2 instrument from the company US instruments was used for the Slingram method and an ARES 1 from the company Gf Instruments was used for the ERT method. Stage 1 took place at full reservoir; stage 2 took place with the reservoir empty. (The reservoir was emptied 3 weeks before the second stage of measurements.) Climatic conditions during both measurement stages were comparable. Temperatures ranged from 10 to $20^{\circ} \mathrm{C}$, and precipitation amounts were within the long-term average. The purpose of repeated measurements with the reservoir empty was to assess the rate of saturation and the seepage curve fluctuation in the dam body. Measured data were reinterpreted in the course of the project MAGIC, in order to refine the boundaries of the seepage segment and test the methodology to determine the water content in earth structures. The layout of the geophysical profiles/survey lines for stage 1 of the survey is shown in Fig. 4.

\section{Electromagnetic survey (Slingram method)}

The aim of the survey was to detect at walking pace, material homogeneities and possible weak zones of the embankment, to be then detailed with higher resolution using ERT. All the results from the three different techniques were cross-compared at the end, to confirm any anomaly, any heterogeneity and any possible seepage pathways.

The results of the measurement using Slingram method are presented in Fig. 5 as a map of apparent resistivity in $\Omega / \mathrm{m}$ instead of electrical conductivity $(\mathrm{mS} / \mathrm{m}$ ) for better comparison with ERT resistivity measurements. The effective skin depths for all the frequencies GEM2 is using are very close to each other, so it was decided to present the results for one frequency for the reservoir full and then for the reservoir empty. The results are presented for a frequency of $47,025 \mathrm{~Hz}$ (Fig. 5) which is a higher frequency setting on the GEM2 in order to reach the upper layers of 


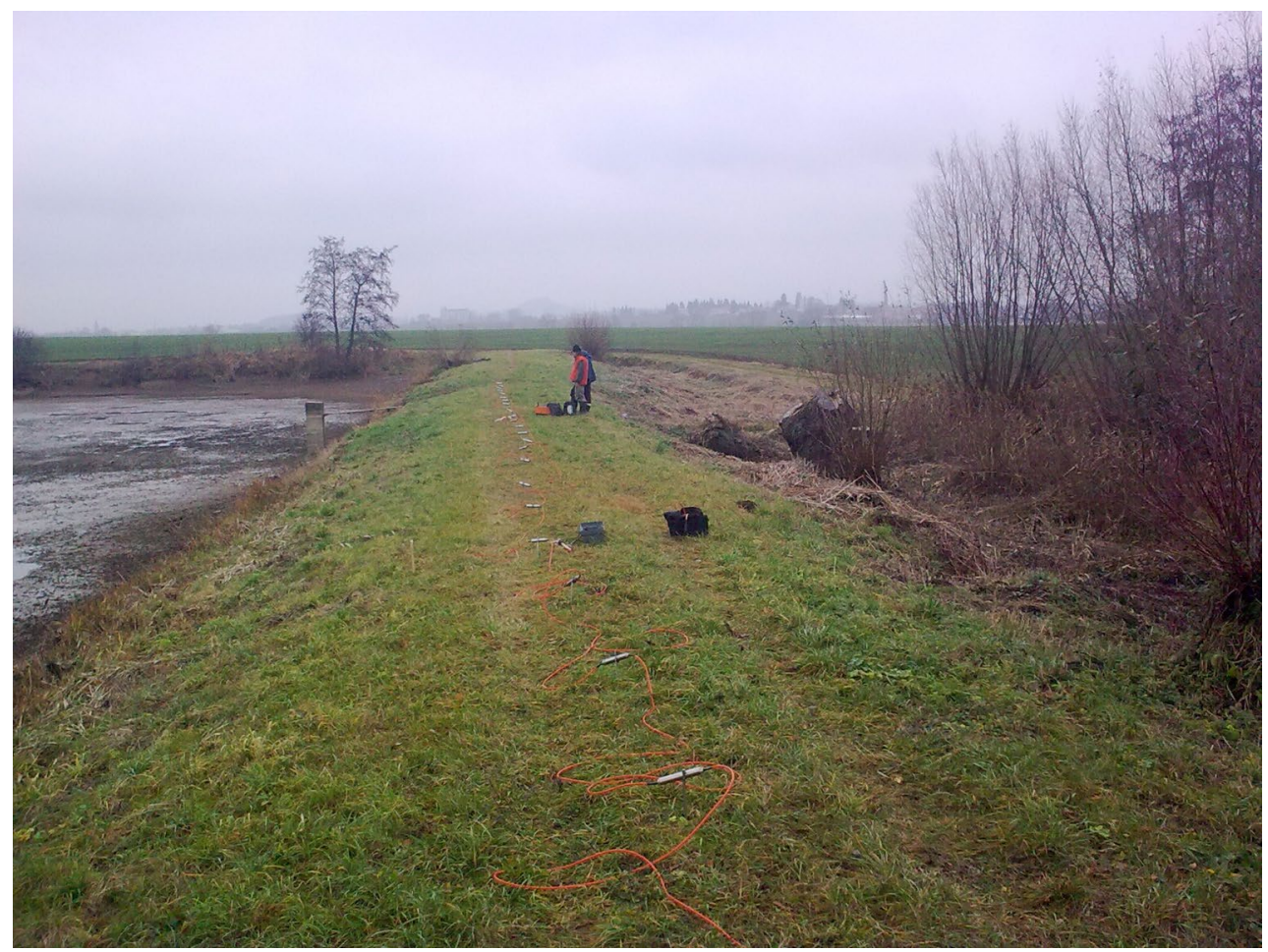

Fig. 3 Embankment picture

the embankment (first meter). The estimation of the depth penetration for different frequencies is based on the "skin depth" parameter (approximately $1 / 2$ of skin depth for resistivity and frequency) and also by past experience. Resistivity values in the dam body most often ranged from 20 to 50 $\Omega / \mathrm{m}$, which corresponds to sandy clay and loam. The dam appeared to show material homogeneity. The exception was the area of the right (southern) keying, where there was an added bench made of construction waste. This area showed slightly increased resistivity values. There was an anomaly area in the middle section of the dam around the bottom outlet (highlighted in Fig. 5 with a square circle), where there was a decrease in the lowest resistivity contour, particularly on the landward slope of the dam. Based on these Slingram measurements, the squared zone could be at risk with a possibility of seepage. The general decrease oin resistivity on the slope was interpreted as increased water saturation for the highest conductivity or lowest resistivity contours (8-24 $\Omega / \mathrm{m}$ ) inside the dam presenting a higher risk of seepage. Hence, the fast-track Slingram measurements identified this area to be selected for further measurements using ERT and SP methods in both stages of the survey (empty and full reservoir).

The Slingram method results were taken at the full reservoir and at empty reservoir and are shown for the frequency of $47,025 \mathrm{~Hz}$ in Fig. 6. The blue line is for the full reservoir and shows lower resistivity meaning higher water saturation.
The red line represents the empty reservoir with higher resistivity. The small decrease in resistivity during the full water capacity (U) possibility means seepage (higher water saturation). The "U" at position $85 \mathrm{~m}$ is a known seepage (observation) near the outlet.

Overall, there was a shift in resistivity on Fig. 6 between the curve representing the full reservoir and the one at empty reservoir. This change can be explained by water saturation fluctuation inside the embankment.

\section{ERT survey}

Measurements using ERT were conducted using the Schlumberger array configuration, which is optimal for mapping sub-horizontal resistivity boundaries. Such a boundary is formed by a saturated zone in the dam body. For seepage investigations, locations where the saturated zone got closer to the surface were particularly checked and also where it directly intersected the landward slope of the dam. It is in such places that usually seepages occur.

Measurements along the longitudinal profile P5 (Fig. 4) were noted every $2 \mathrm{~m}$, and every $1 \mathrm{~m}$ for the profiles across the width. A comparison of measurements for full and empty reservoir was carried out, especially where the height of the saturated zone (or generally the water content in the dam body) fluctuates rapidly depending on the water level in the reservoir. The occurrence of permeable zones due to 
Fig. 4 Map of the electromagnetic, ERT and SP profiles along Vitineves reservoir dam

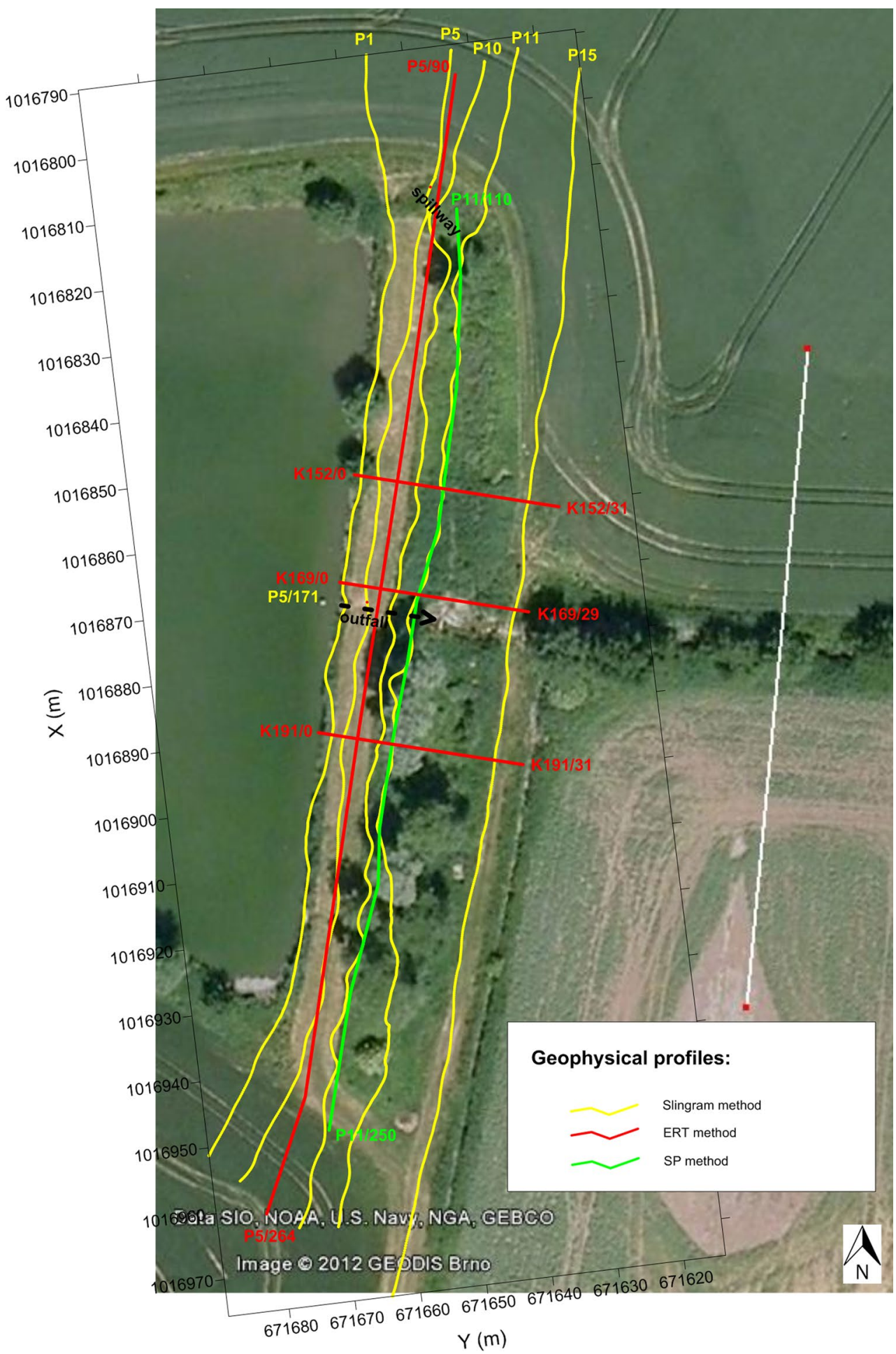

material inhomogeneity, cracks, construction joints, which often lead to seepages, were expected where the response to a change of water level in the reservoir was rapid.

Examples of resistivity sections according to ERT are shown in Fig. 7. The profiles were obtained using the commercial software Res2DInv. The results presented were inverted using the standard method of inversion (L2-norm approach) after the fifth iteration. For all sections results, an RMS model error below $4.5 \%$ was obtained. No corrections due to temperature changes in the dam body were made as both measurement stages took place during a short time interval of 10 weeks and the autumn climatic conditions did not exhibit influential variations. In some sections, there was a highlighted contour of resistivity within a range of $15-20$ 


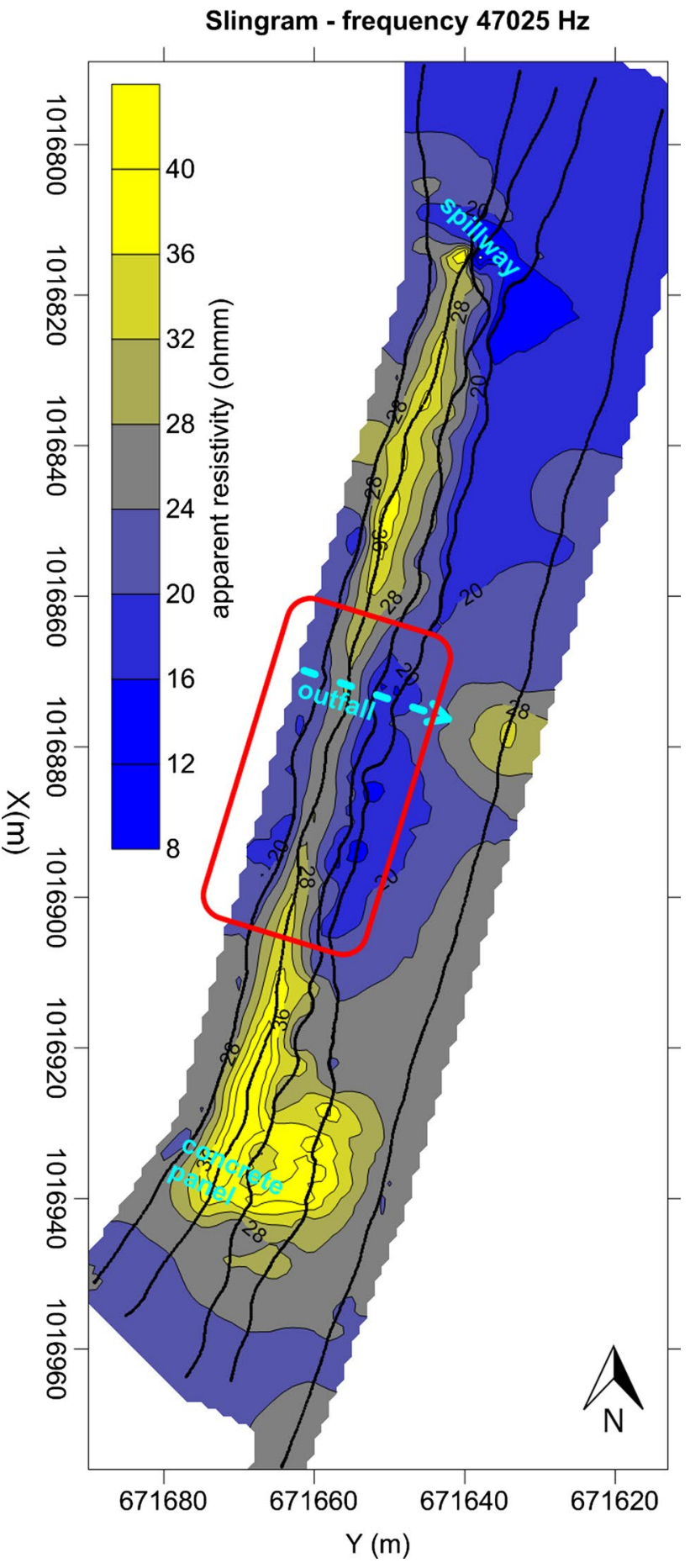

Fig. 5 Slingram method - map of apparent resistivity

$\Omega / \mathrm{m}$. This contour could be related to the presence of seepage based on observations of known seepages in the dam. The interpreted course of the seepage curve is highlighted as a white dashed line on Fig. 8. The upper part of the figure shows a longitudinal profile P5 along the dam body at full and empty reservoir. In the middle part of the dam (interval between 154 and $200 \mathrm{~m}$ ), there is an obvious area, where the interpreted course of the seepage curve is apparently closer to the surface and where the curve at empty reservoir rapidly dropped. The area is highlighted with a yellow rectangle and is considered at risk in terms of occurrence of seepages.

Two cross sections through the dam body are presented on Fig. 8. Profile K152 is located beyond the interpreted area at risk with seepages; profile K169 is located in the middle of the area. The difference in the course of the seepage curve is obvious. For profile K169, at full reservoir, the seepage curve intersects the slope surface of the dam approximately in the middle. At empty reservoir, a rapid drop of the curve below the ground surface occurred. Near the profile at the same level, a long-term seepage was observed. On the profile K152, the seepage curve was safely located below the ground level, and the response of the discharged reservoir was small. The dam remained saturated at normal level; a decrease in water level took place very slowly. The cross sections confirmed the interpretation of the risk posed by the area of the dam spotted from the longitudinal profile. Overall, the ERT results highlight an increase in the water saturation during the full water stage on the part of the dam flank investigated.

\section{Self-potential (SP) survey}

Measurement of the natural electric potentials using SP method was intended to confirm the interpretation of risk posed in the middle area of the dam. Measurements were conducted using non-polarisable electrodes with intervals of $5 \mathrm{~m}$, using potential method ( $\mathrm{N}$ electrode was stable near the right keying of the dam). The two non-polarised $\mathrm{Cu}$ / $\mathrm{CuSO}_{4}$ electrodes were first placed together at the soil surface to zero the potential. One electrode was taken as origin of the profile and kept static as a common reference point for all measurements. The second electrode was moved along the profile to take measurements every $5 \mathrm{~m}$ over the length of the dam. The results at both stages for the profile P11 taken at the dam toe are shown in Fig. 9 in the form of graphs of electric potential. This profile can be compared with the ERT profile P5 showed on the same figure in order to provide an interpretation of seepages as the two profiles were parallel in orientation. Measurements at full reservoir showed several positive anomalies in the middle part of the dam. The most significant anomaly was located between 165 and $170 \mathrm{~m}$ and reached up to $50 \mathrm{mV}$ also confirmed on the P5 and K169 ERT profiles. Less significant anomalies were located at 185, 200 and $215 \mathrm{~m}$ with amplitudes ranging from 10 to $15 \mathrm{mV}$. Measurements at the empty reservoir showed a decrease in all anomalies below $10 \mathrm{mV}$. The local negative anomaly at $225 \mathrm{~m}$ was probably a measurement error. The measured positive anomalies showed shallow seepages that didn't occur at empty reservoir. Water seeping through a porous environment usually shows a loss of dissolved 


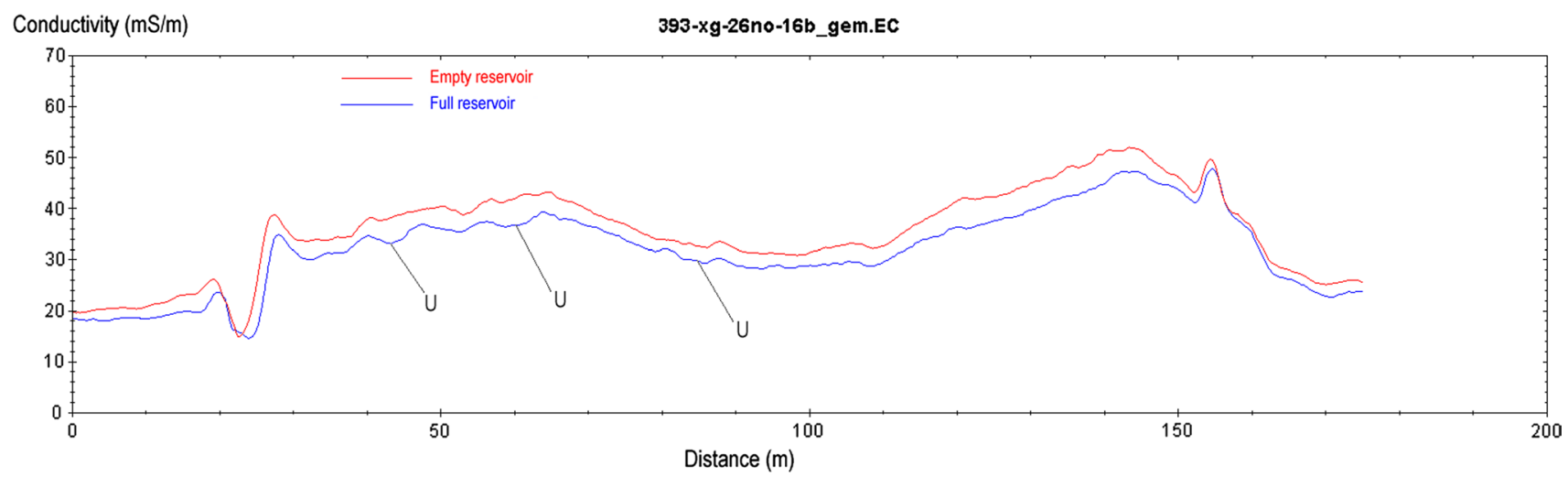

Fig. 6 Slingram method—comparison full and empty reservoir

Fig. 7 ERT method P5 profile with anomalous section

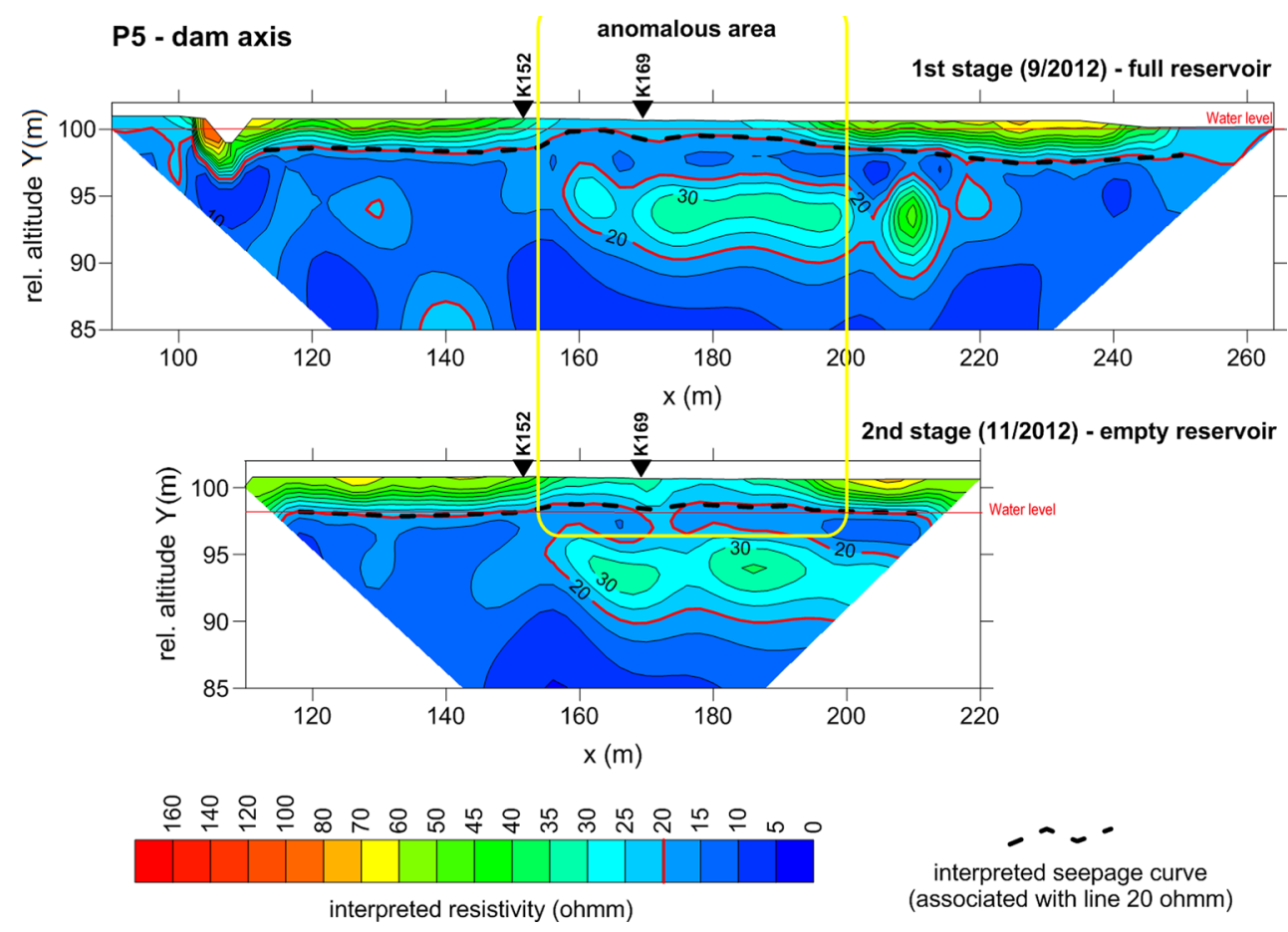

negative particles (anions). They are captured on the walls of the soil pores. Near an outflow there is the formation of positive electric potential.

For deeper and long-term seepages, situation may be different. In the zone above the seepage, a local minima of SP potential could occur due to the capture of anions by the soil. A negative anomaly could be formed as the environment is actually enriched with captured anions. All measurements were carried out with the same GEM-2 equipment, using the same location and the same procedure. The seepage has also been confirmed by visual observations. It is coincidental that the seepage anonmy is so close to the outfall. The pipe did not have a strong influence on the readings, as the signal at the empty reservoir stage would have exhibited a higher peak than the one displayed on Fig. 9. The difference between the potential peaks at full reservoir and at empty reservoir is only due to differences in water saturation.

Finally, Fig. 10 shows all the different profiles carried out (Slingram, P5-ERT, SP measurements etc...) with the location of all geophysical and visual anomalies. For instance, it can be seen on the picture that the microgravity techniques detected the presence of anomaly between local scale positions $(X=1016880 \mathrm{~m}, Y=671,652)$ and $(X=1016860 \mathrm{~m}$, $Y=671647 \mathrm{~m})$ and confirmed the location of the seepage detected by the self-potential technique at $(X=1016870 \mathrm{~m}$, $Y=671645 \mathrm{~m})$. The self-potential anomalies signatures are represented as red lozenges on Fig. 10 and correspond to the red triangles (interpreted seepage) on Fig. 9. Similarly, the 


\section{Profil K152 - 1. 1st stage - Full reservoir}

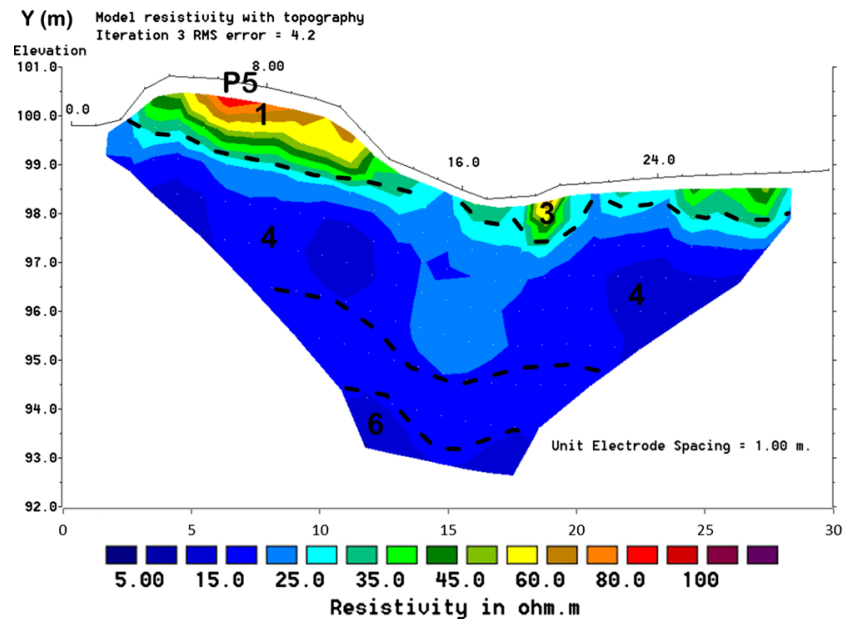

Profil K169 - 1. 1st stage - Full reservoir

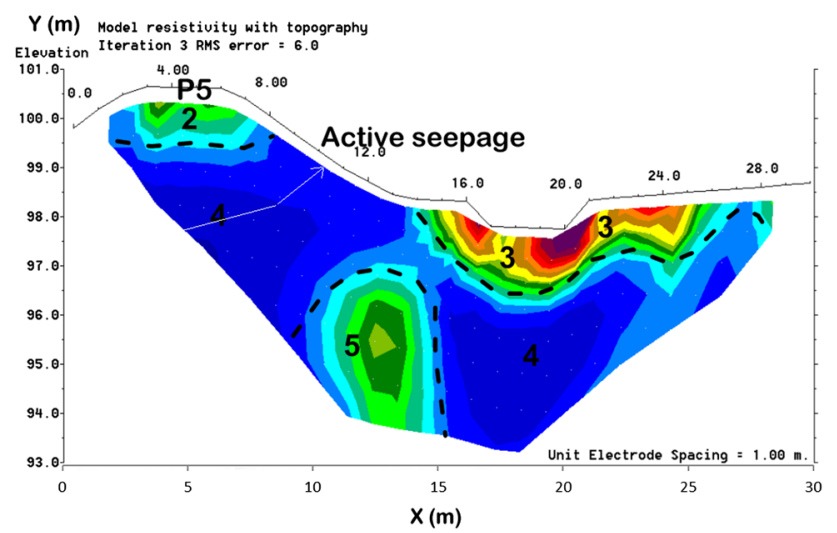

Fig. 8 ERT cross-section profiles for full and empty reservoir

slingram method gave the same anomaly boundary between the positions indicated by the transversal black dotted lines. In the legend, the local shape down of resistivity during full reservoir obtained by ERT techniques is indicating a decrease in resistivity at full reservoir compared with empty reservoir. These low-resistivity zones are expected to have relative higher water content and to be prone to seepage.

Hence, the ERT anomaly detected at the distance $155-200 \mathrm{~m}$ of the profile in Fig. 7 corresponds to the selfpotential anomaly detected at the position $165-170 \mathrm{~m}$ ) Fig. 9, which is the main seepage area (also clearly visible on the perpendicular cross section K169 (Fig. 8), which is near the outlet section on Fig. 10 as explained previously.

\section{Czech reservoir assessment recommendations}

The aim of the geophysical survey on the dam of Vitiněves reservoir was to assess the material homogeneity of the dam and to determine the long-term prediction of seepages that

\section{Profil K152 - 2nd stage - Empty reservoir}

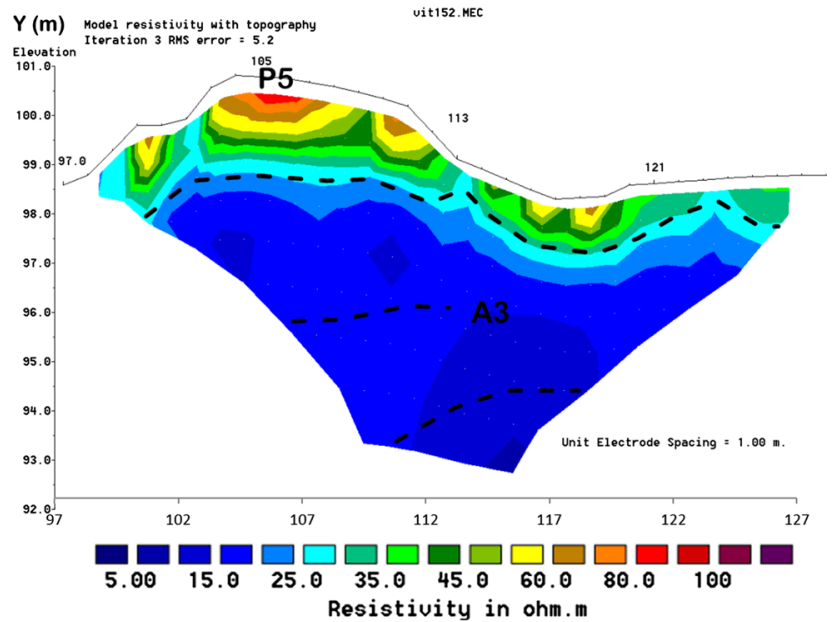

Profil K169 - 2nd stage - Empty reservoir

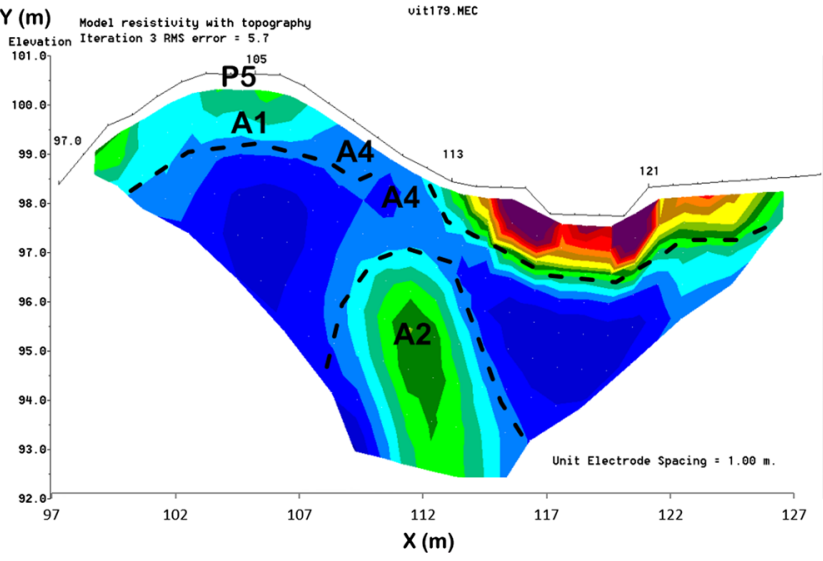

could occur. The results were used to design remedial measures to reduce seepages. The survey was conducted using Slingram, ERT and SP methods in two stages. Stage 1 took place at an operating level in the reservoir (full), and stage 2 took place when the reservoir was empty.

Slingram method was used to search for the area of decreased resistivity in the middle part of the dam and indicated the risk of anomalous water saturation inside the dam body. Measurements using ERT and SP methods in both stages of the survey were carried out. The difference in resistivity or electric potentials at full and empty reservoir allowed better understanding of the interpretation of the path of the seepage curve through the dam body and to a more accurate understanding of the occurrence of seepages. All methods have confirmed the occurrence of anomalous area in the middle part of the dam in the segment between 154 and $200 \mathrm{~m}$ (or even $215 \mathrm{~m}$ according to SP). This segment was recommended to be subjected to remedial measures to reduce the risk of seepages. A pipe that would safely transfer 

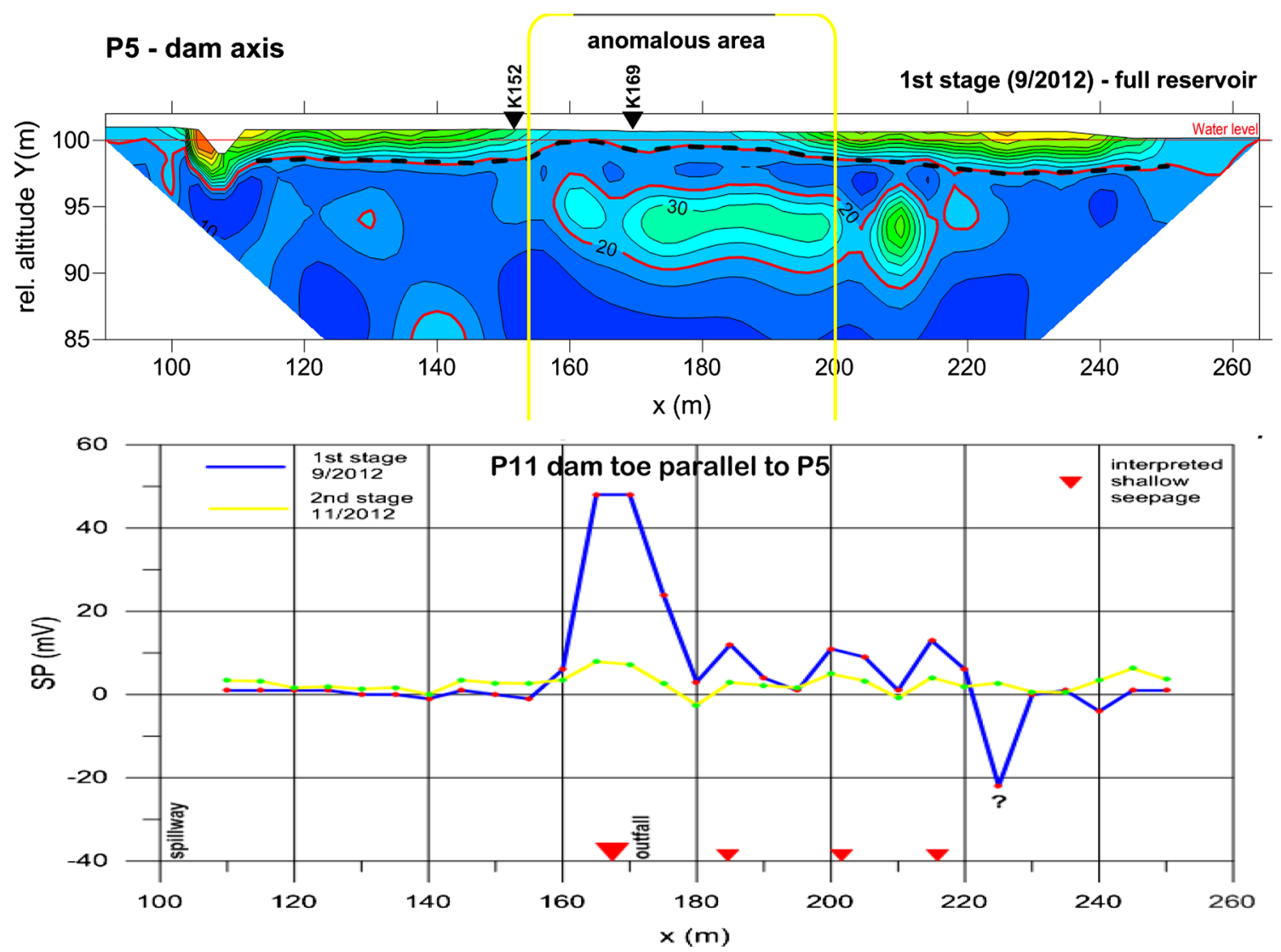

Fig. 9 Graphs of SP potential with ERT profile. Interpretation of shallow seepages

shallow seepages to the subsoil beyond the dam heel was suggested as a suitable method of remediation.

\section{Conclusion}

By using Slingram method, electrical resistivity tomography (ERT) method and self-potential (SP) method, strategic information could be obtained. The integrity of the embankment was never compromised as these techniques are all non-intrusive. These techniques are fast and more economical than traditional trenching or borehole investigations. With the Slingram method (GEM2 apparatus), no anomalies were missed in the Czech reservoir, which could have led to catastrophic results in both social and economic terms. Full appraisal was carried out by the application of high-resolution techniques at the specified locations with ERT and SP for potential seepage.

To conclude, the electromagnetic survey (EM) with the Slingram method was used as a fast technique to identify weak spots related to areas of high variability in conductivity that may be an indication of erosion leading to piping.
This method was effective and fast and gave a basic description of material homogeneity and helped in the identification of the problematic zones. Self-potential (SP) and ERT provided the best interpretation of seepages with higher resolution. Repeated geoelectrical measurement revealed the relative changes of water saturation in the selected reservoir embankment.

The interpretation of the geophysical measurements was made more accurate by comparing the results from the three geophysical techniques. The advantage of using all of them together was to confirm the same anomalies and the same findings (seepage and erosion).

By using all three techniques together, the assessor has a degree of certainty and confidence in the results meaning that the most appropriate and economical solution can be applied by reducing the need for more invasive ground investigations.

The value of using these three combined techniques cannot be underestimated for both flood prevention and damage remediation and could be beneficial as normal practice for the assessment of flood defences. 
Fig. 10 Complementary figure of all techniques used
Local shape down of resistivity during full reservoir

basic SLINGRAM anomaly

(boundary of anomaly from Fig. 3)

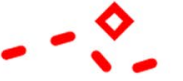

anomaly of microgravimetry (lower density material, dig over section )

SP anomaly (risk of seepage)

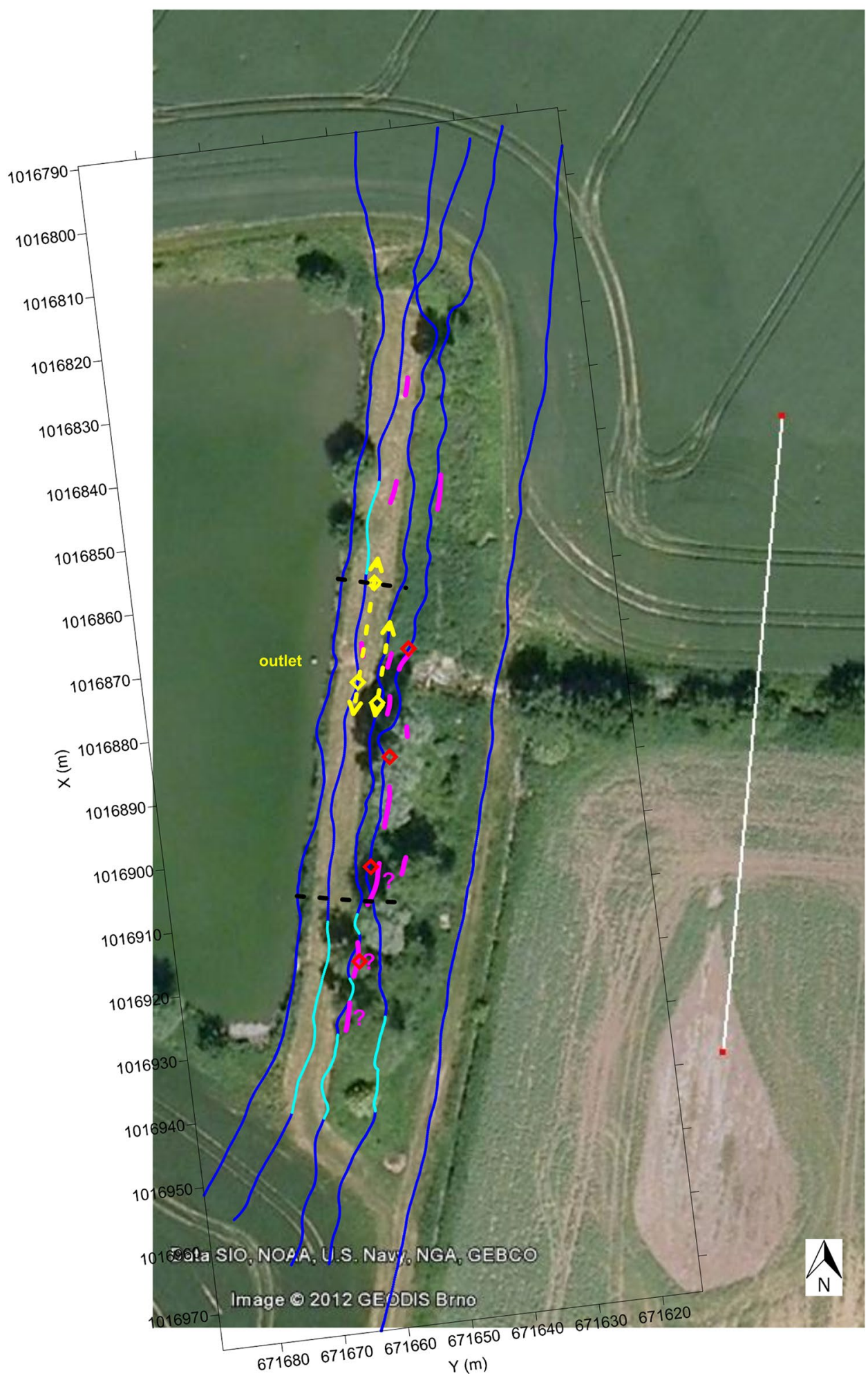


Acknowledgements The authors wish to acknowledge the support of the European Commission via the Marie Curie IAPP project MAGIC-Monitoring systems to Assess Geotechnical Infrastructure subjected to Climatic hazards (FP7-PEOPLE-2012-IAPP-324426) for the Czech Republic surveys and to the Impact Acceleration Account from Strathclyde University and the ICE enabling fund for their support for the research on river and coastal flood defences assessment using Geophysics.

Open Access This article is distributed under the terms of the Creative Commons Attribution 4.0 International License (http://creativeco mmons.org/licenses/by/4.0/), which permits unrestricted use, distribution, and reproduction in any medium, provided you give appropriate credit to the original author(s) and the source, provide a link to the Creative Commons license, and indicate if changes were made.

\section{References}

Antoine R, Fauchard C, Fargier Y, Durand E (2015) Detection of leakage areas in an earth embankment from GPR measurements and permeability logging. Int J Geophys 2015:1-9

Benes V, Tesař M, Boukalová Z (2011) Repeated geophysical measurement-the basic principle of the GMS methodology used to inspect the condition of flood control dikes. River Basin Management 2011 (25-27 May, Riverside, California, USA)

Bergamo P, Dashwood B, Uhlemann S, Swift Chambers JE, Gunn DA, Donohue S (2016) Time-lapse monitoring of fluid-induced geophysical property variations within an unstable earthwork using P-wave refraction. Geophysics 81(4):17-27

Bièvre G, Lacroix P, Laurent O, David G, Guy M, Fargier Y (2017) Integration of geotechnical and geophysical techniques for the characterization of a small earth-filled canal dyke and the localization of water leakage. J Appl Geophys 139:1-15

Bolève A, Janod F, Revil A, Lafon A, Fry JJ (2011) Localization and quantification of leakages in dams using time-lapse selfpotential measurements associated with salt tracer injection. $\mathrm{J}$ Hydrol 403(3-4):242-252

Chao C et al (2006) Integrated geophysical techniques in detecting hidden dangers in river embankments. J Environ Eng Geophys 11:83-94. https://doi.org/10.2113/JEEG11.2.83

Cho IK, Yeom JY (2007) Cross line resistivity tomography for the delineation of anomalous seepage pathways in an embankment dam. Geophysics 72:G31. https://doi.org/10.1190/1.2435200

Di Prinzio M, Bittelli M, Castellarina A, Rossi P (2010) Application of GPR to the monitoring of river embankments. J Appl Geophys 71(2-3):53-61

Dyer MR, Utili S, and Zielinski M (2009) Field study into fine desiccation fissuring at Thorngumbald. In: ICE Proceedings of water management, issue WM3, pp 221-232

Fargier Y, Palma Lopes S, Fauchard C, François D, Côte P (2014) DC-electrical resistivity imaging for embankment dike investigation: a 3D extended normalisation approach. J Appl Geophys 103:245-256

Fauchard C, Meriaux P (2007) Geophysical and geotechnical methods for diagnosing flood protection dikes-guide for implementation $\mathrm{n}$ and interpretation. Editions Quae. ISBN: 978-2-7592-0035-1

Griffiths DH, Barker RD (1993) Two-dimensional resistivity imaging and modelling in areas of complex geology. J Appl Geophys 29:211-226

Hadley LM (1983) Geophysical method of evaluating existing earth embankments. Bull Assoc Eng Geol 20(3):289-295
Ikard SJ, Revil A, Jardani A, Woodruff WF, Parekh M, Mooney M (2012) Saline pulse test monitoring with the self-potential method to non-intrusively determine the velocity of the pore water in leaking areas of earth dams and embankments. Water Resour Res 48(W04201):1-17. https://doi.org/10.1029/2010WR0102

Ikard SJ, Revil A, Schmutz M, Karaoulis M, Jardani A, Mooney M (2014) Characterization of focused seepage through an earthfill dam using geoelectrical methods. Groundwater 52(6):952-965

Jones G, Sentenac P, Zielinski M (2014) Fissure detection using 2-D and 3-D electrical resistivity tomography on a flood embankment. J Appl Geophys 106:196-211

Lin CP, Hung YC, Wu PL, Yu ZH (2014) Performance of 2-D ERT in investigation of abnormal seepage: a case study at the Hsin-Shan earth dam in Taiwan. J Environ Eng Geophys 19(2):101-112

McDowell PW et al (2002) Geophysics in engineering investigations. Constr Ind Res Inf Assoc CIRIA C562:252

Minsley BJ, Burton BL, Ikard S, Powers MH (2011) Hydrogeophysical investigations at hidden dam, Raymond, California. J Environ Eng Geophys 16(4):145-164

Mydlikovski R, Beziuk G, Szynkiewicz A (2007) Detection of inhomogeneities in structure of flood embankments by means of D.C. resistivity, GPR and frequency electromagnetic method measurements. Acta Geodyn Geomater 4(148):83-88

Nguyen $F$ et al (2005) Image processing of $2 D$ resistivity data for imaging faults. J Appl Geophys 57(4):260-277

Niederleithinger E, Weller A, Lewis R (2012) Evaluation of geophysical techniques for dike inspection. JEEG 17(4):185-195

Perri MT, Boaga J, Bersan S, Cassiani G, Cola S, Deiana R, Simonini P, Patti S (2014) River embankment characterization: the joint use of geophysical and geotechnical techniques. J Appl Geophys 110:5-22

Planès T et al (2016) Time-lapse monitoring of internal erosion in earthen dams and levees using ambient seismic noise. Géotechnique 66(4):301-312

Reynolds JM (2011) An introduction to applied and environmental geophysics, 2nd edn. Wiley, Chichester, p 712

Rittgers JB, Revil A, Planes T (2015) 4-D imaging of seepage in earthen embankments with time-lapse inversion of self-potential data constrained by acoustic emissions localization. Geophys J Int 200(2):758-772

Sentenac P, Jones G, Zielinski M, Tarantino A (2013) An approach for the geophysical assessment of fissuring of estuary and river flood embankments: validation against two case studies in England and Scotland. Environ Earth Sci 69(6):1939-1949

Sentenac P, Benes V, Budinski V, Keenan H, Baron R (2017) Post flooding damage assessment of a historical pond and earth dam by non-invasive geophysical techniques. J Appl Geophys 146:138-148

Seokhoon Oh (2012) Electrical resistivity response due to variation in embankment shape and reservoir levels. Environ Earth Sci 65(3):571-579

Simon FX, Sarris A, Thiesson J, Tabbagh A (2015) Mapping of quadrature magnetic susceptibility/magnetic viscosity of soils by using multi-frequency EMI. J Appl Geophys 120:36-47

Sjödahl P, Dahlin T, Zhou B (2006) 2.5 D resistivity modeling of embankment dams to assess influence from geometry and material properties. Geophysics 71(3):107-114. https://doi. org/10.1190/1.2198217

Sjödahl P, Dahlin T, Johansson S (2010) Using the resistivity method for leakage detection in a blind test at the Røssvatn embankment dam test facility in Norway. Bull Eng Geol Env 69(4):643-658

Slater L (2007) Near surface electrical characterization of hydraulic conductivity from petrophysical properties to aquifer geometries, a review. Surv Geophys 28:169-197 
Takahashi T, Yamamoto T (2010) An attempt at soil profiling on a river embankment using geophysical data. Explor Geophys 41(1):102-108

Utili S, Castellanza R. Galli A, Sentenac P (2014) Novel approach for health monitoring of earthen embankments. J Geotech Geoenviron Eng 141(3):1-19

Viganotti M, Jackson R, Krahn H, Dyer M (2013) Geometric and frequency EMI sounding of estuarine earthen flood defence embankments in Ireland using 1D inversion models. J Appl Geophys 92:110-120

Weller A, Lewis R, Canh T, Möller M, Scholz B (2014) Geotechnical and geophysical long-term monitoring at a levee of Red River in Vietnam. J Environ Eng Geophys 19(3):183-192 